Brief

Bommunioation

Submitted: 14 Dec 2017

Online: 28 Jun 2018
Accepted: 16 Apr 2018

\section{Antimicrobial Susceptibility Patterns of an Emerging Multidrug Resistant Nosocomial Pathogen: Acinetobacter baumannii}

\author{
Rachna TeWARI ${ }^{1}$, Deepti Chopra ${ }^{2}$, Rushna WAZAHAT ${ }^{1}$, Shreya \\ Dhingra ${ }^{1}$, Mridu DudeJA ${ }^{1}$
}

1 Department of Microbiology, HIMSR, Jamia Hamdard, New Delhi-62, India

2 Department of Pharmacology, Government Institute of Medical Sciences, Kasna, Greater Noida, Uttar Pradesh-201310, India

To cite this article: Tewari R, Chopra D, Wazahat R, Dhingra S, Dudeja M. Antimicrobial susceptibility patterns of an emerging multidrug resistant nosocomial pathogen: Acinetobacter baumannii. Malays J Med Sci. 2018;25(3):129134. https://doi.org/10.21315/mjms2018.25.3.13

To link to this article: https://doi.org/10.21315/mjms2018.25.3.13

Multidrug-resistant (MDR) Acinetobacter baumannii (A. baumannii) bacterium, a nosocomial pathogen associated with a high mortality rate and limited therapeutic options have emerged as a serious problem throughout the world. The present study aimed to assess the current levels of antibiotic susceptibility among the isolates of Acinetobacter species. The sensitivity patterns were analysed from various clinical specimens obtained from both in-patients and outpatients of a teaching hospital. Isolation was performed on $5 \%$ sheep blood agar and MacConkey agar. Urine samples were inoculated into CLED agar. Antibiotic susceptibility was performed by the disc diffusion method. A total of 16,452 samples were collected. The total number of samples positive for Acinetobacter species was $67(0.4 \%)$. The highest number of isolates 26 (38.8\%) were obtained from urine. Majority $80.3 \%$ of the isolates exhibited resistance to three or more classes of antibiotics. All isolates were susceptible to colistin (100\%). The susceptibility rate of $A$. baumannii isolates was $80 \%$ for tigecycline and $53.3 \%$ for carbapenem. Combination therapies including colistin and tigecycline seem to be the rational treatment for MDR $A$. baumannii until new alternatives come forward.

Keywords: Acinetobacter, multidrug resistance, antibiotics, colistin, tigecycline, carbapenems

\section{Introduction}

Acinetobacter are aerobic, gram negative non-fermenting, non-fastidious, non-motile, catalase-positive, and oxidase negative coccobacilli that prefer a moist environment (1). The genus Acinetobacter has taken more and more imperative place as an opportunistic, difficult-to-treat pathogen causing nosocomial infections, though community acquired infections have also been reported. Acinetobacter is accredited as one of the six intricate pathogens "ESKAPE" (Enterococcus faecium, Staphylococcus aureus, Klebsiella pneumoniae, Acinetobacter baumanni, Pseudomonas aeruginosa, and Enterobacter species) to emphasise that they escape the lethal action of antibiotics (2). Numerous studies have documented that Acinetobacter species have a noteworthy capacity for long-term survival (even in dry conditions) on various equipments like respirators and other inanimate surfaces in the hospital environment including telephone handles, door pushplates, patient charts, tabletops, hospital floor, hospital sink traps, bed linen, etc (3). The most important species of this organism is Acinetobacter baumannii (A. baumannii) causing most of the reported outbreaks.

During the course of time Acinetobacter species have acquired resistance to almost all available antimicrobial agents. The spectrum 
of antibiotic resistance of these organisms makes them a threat in hospital environment, as documented by recurring outbreaks and has created major challenges for healthcare management worldwide (4). The appearance of resistant Acinetobacter species is attributed to both selective pressure exerted by the use of broad spectrum antimicrobials and health care associated transmission of drug-resistant strains among patients (4). A number of acquired mechanisms of resistance including production of extended spectrum beta-lactamase enzymes, modification enzymes against aminoglycosides, altered binding sites for quinolones, and a variety of efflux mechanisms result in significant challenges for the clinician to select an appropriate empirical antimicrobial agent (4).

Thus, the aim of the present study was to assess the current levels of antimicrobial susceptibility among the clinical isolates of Acinetobacter species recovered from different clinical specimens obtained from in-patients and out-patient department of a teaching hospital.

\section{Materials and Methods}

The present retrospective study was conducted in a 470-bedded teaching hospital, in Delhi, India by the Department of Microbiology and Department of Pharmacology over a 2-year period (January 2013-December 2015) after obtaining approval from the internal review board. Various clinical samples collected aseptically and processed during routine diagnostic work up from both inpatients and patients visiting the outpatient departments were analysed.

Isolation of Acinetobacter species was performed on 5\% sheep blood agar and MacConkey agar. Urine samples were inoculated into CLED agar and identification of clinical isolates was performed by grams staining, colony morphology and biochemical reactions. Acinetobacter species was identified as non-lactose fermenting, non-motile, oxidase negative, gram negative coccobacilli colonies and biochemical reactions. Species differentiation was done on the basis of glucose oxidation, gelatin hydrolysis, haemolysis, growth at $35{ }^{\circ} \mathrm{C}$ and $44{ }^{\circ} \mathrm{C}$ and assimilation tests (5).

Identification was confirmed by an automated system, VITEK 2 (BioMerieux, France). VITEK 2 system uses the principles of Advanced Colorimetry. Identification of all isolates was executed with a pure overnight subculture as recommended by the manufacturer. Results are given as per the database in instrument, which is regularly updated by the manufacturer.

Antibiotic susceptibility was performed by the Kirby Bauer disc diffusion method. The bacterial suspension of each sample was made and compared with 0.5 McFarland turbidity standard. The cartridges containing antimicrobial susceptibility discs (Himedia, Mumbai) were kept at temperature between $4{ }^{\circ} \mathrm{C}$ and $-20{ }^{\circ} \mathrm{C}$, and used after incubation at room temperature. Mueller-Hinton agar plates were inoculated and incubated at $35^{\circ} \mathrm{C}$ for $18 \mathrm{~h}$, and the diameter of the zones of inhibition were measured and interpreted as recommended by Clinical and Laboratory Standards Institute (CLSI) 2010 guidelines (6).

The antibiotics tested were Ampicillin (10 $\mu \mathrm{g})$, Ampicillin/Sulbactam (10/10 $\mu \mathrm{g})$, Co-amoxiclav $(20 / 10 \mu g)$ Amikacin (30 $\mu \mathrm{g})$, Ceftazidime (30 $\mu \mathrm{g})$, Sulbactam/cefaperazone $(75 / 30 \mu \mathrm{g})$, Ciprofloxacin $(5 \mu \mathrm{g})$, Gentamicin $(10 \mu \mathrm{g})$, Meropenem $(30 \mu \mathrm{g})$, Imipenem $(10 \mu \mathrm{g})$, Ofloxacin $\quad(5 \mu \mathrm{g})$, Piperacillin/Tazobactam $(110 \mu \mathrm{g})$, Norfloxacin (10 $\mu \mathrm{g})$, Nalidixic acid (30 $\mu \mathrm{g})$, Ticarcillin $(75 \mu \mathrm{g})$, Piperacillin $(100 \mu \mathrm{g})$, sulfamethoxazole-trimethoprime $(1.25 / 23.7 \mu \mathrm{g})$, Tigecycline $(15 \mu \mathrm{g})$, Clindamycin $(2 \mu \mathrm{g})$, Cefepime $(30 \mu \mathrm{g})$, Nitrofurantoin $(300 \mu \mathrm{g})$, Aztreonam (30 $\mu \mathrm{g})$, and Colistin (110 $\mu \mathrm{g})$. Antibiotic discs were obtained from Himedia, Mumbai, India. Escherichia coli ATCC 25922 and Pseudomonas aeruginosa ATCC 27853 were used as control strains for quality control of media and antibiotic discs.

All isolates of Acinetobacter resistant to three or more classes of antibiotics were considered as multidrug resistant (MDR).

\section{Results}

\section{Bacterial Isolates}

During the study period, a total of 16,452 samples were collected in the bacteriology laboratory. The total number of samples positive for Acinetobacter species was 67 (0.4\%). The frequency of Acinetobacter species in male and female patients was $27(40.3 \%)$ and 40 (59.7\%), respectively. Mean age of the patients being $63 \pm$ 0.8 years. 


\section{Types of Clinical Specimens}

The isolates were predominantly recovered from urine samples (38.8\%) followed by pus/ wound swab cultures (22.3\%). The types of clinical specimens are depicted in the Table 1.

Table 1. Types of clinical specimens

\begin{tabular}{lc}
\multicolumn{1}{c}{ Clinical specimen } & Number (\%) \\
Urine & $26(38.8 \%)$ \\
Pus/ wound culture & $15(22.3 \%)$ \\
Blood & $13(19.4 \%)$ \\
Respiratory tract & $10(14.9 \%)$ \\
(Sputum, Bronchial lavage, & \\
Endotracheal tube secretion) & \\
Others & $3(4.5 \%)$ \\
\hline
\end{tabular}

\section{Antibiotic Susceptibility Pattern}

Acinetobacter species showed high antibiotic resistance rate, with great percentage (80.3\%) of the isolates exhibiting resistance to three or more classes of antibiotics. Urine samples showed the greatest yield of resistant A. baumannii. Only $7.8 \%$ of the isolates were sensitive to all the drugs.

The results of antimicrobial susceptibility tests showed that most (86.6\%) of the $A$. baumannii isolates were MDR strains i.e., resistant to three or more classes of antibiotics while $13.3 \%$ were resistant to two classes of antibiotics (Figure 1). All the A. baumannii isolates were sensitive to colistin (100\%) while $80 \%$ were sensitive to tigecycline and 53.3 $\%$ were sensitive to carbapenems (Table 2). Antibiotic susceptibility pattern of isolates of A. baumannii group differed from the nonbaumannii Acinetobacter group (Table 2).

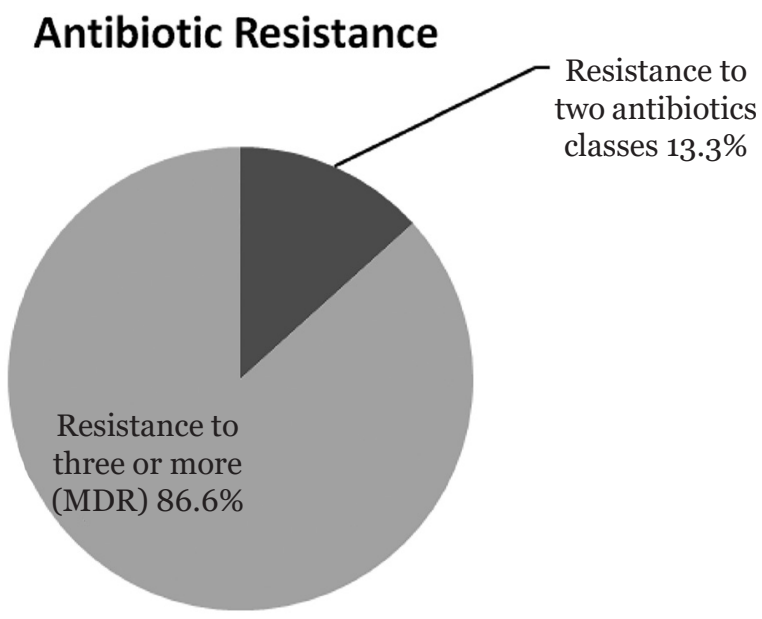

Figure 1. Percentage of Acinetobacter baumannii resistant to various numbers of antibiotics classes

\section{Discussion}

Antimicrobial resistance among Acinetobacter species has increased at a disquieting rate leading to increased morbidity, mortality and treatment costs in Intensive Care

Table 2. Antibiotic susceptibility of Acinetobacter species

\begin{tabular}{lcc} 
Antibiotic & $\begin{array}{c}\text { Acinetobacter baumannii } \\
\text { Sensitivity } \boldsymbol{n}(\%)\end{array}$ & $\begin{array}{c}\text { non-baumannii Acinetobacter } \\
\text { Sensitivity } \boldsymbol{n}(\%)\end{array}$ \\
Colistin & $45(100 \%)$ & $22(100 \%)$ \\
Tigecycline & $36(80 \%)$ & $12(54.5 \%)$ \\
Carbapenems & $24(53.3 \%)$ & $16(72.7 \%)$ \\
Cefoperazone/ sulbactam & $21(46.6 \%)$ & $10(45.4 \%)$ \\
Cefepime & $18(40 \%)$ & $11(50 \%)$ \\
Piperacillin /tazobactam & $15(33.3 \%)$ & $10(45.4 \%)$ \\
Amikacin & $15(33.3 \%)$ & $9(40.9 \%)$ \\
Piperacillin & $14(31.1 \%)$ & $10(45.4 \%)$ \\
Cotrimoxazole & $12(26.7 \%)$ & $9(40.9 \%)$ \\
Ciprofloxacin & $9(20 \%)$ & $9(40.9 \%)$ \\
Nalidixic acid & $9(20 \%)$ & $9(40.9 \%)$ \\
Nitrofurantoin & $3(6.6 \%)$ & $4(18.2 \%)$ \\
Amoxiclav & $3(6.6 \%)$ & $8(36.4 \%)$ \\
\hline
\end{tabular}


Units (ICU). Definitions of multidrug-resistant Acinetobacter species vary, the most widespread being isolates showing either carbapenem resistance or resistance to more than three classes of antimicrobials (7).

In the present study, there was predominance of isolates from urine samples. This finding is similar to various studies from India and other countries, demonstrating predominance of isolation of Acinetobacter from urine specimens $(8,9)$. In contrast, some studies have shown respiratory secretions as the most common specimen from which Acinetobacter are isolated $(9,10,11)$. Isolation rate from blood in this study was $19.4 \%$ whereas different studies have reported isolation rates ranging from $7 \%-$ $25 \%(8,10,12)$.

The resistance pattern of the $A$. baumannii isolates has varied according to the geographic location. In India, there has been an increasing trend towards multidrug resistant Acinetobacter. In present study, overall 80.3\% of the Acinetobacter species and $86.6 \%$ of the $A$. baumannii isolates were MDR. Likewise, other parts of the world have also reported increasing MDR trend. Nazmul et al. (12) reported $85 \%$ MDR Acinetobacter isolates from Malaysia wherein Vakili et al. (11) reported 95\% MDR A. baumannii isolates from Iran. Similar to this study, the percentage of isolates showing MDR were highest from urine $(8,12)$.

In the present study, the sensitivity of the isolates (both A. baumannii and non-baumannii Acinetobacter species) to piperacillin was low. Further, studies conducted in other parts of the world between 2012 and 2014 reported a high prevalence of resistance to piperacillin. Nazmul et al. (12) reported $77.5 \%$ resistance to piperacillin whereas, Shakibaie et al. (13) reported $100 \%$ resistance. A recent study from India by Gupta et al. (14) reported 55\% resistance to piperacillin.

The result of the present study showed an increasing trend for development of resistance of the A. baumannii species towards the piperacillin/tazobactam combination. Only $33.3 \%$ were sensitive to piperacillin/tazobactam combination. This is in accordance with studies from India and other countries, which also reported a high resistance rate of $A$. baumannii isolates to piperacillin/tazobactam combination $(10,15)$.

Resistance to carbapenems has also increased. A few earlier studies from India have reported low resistant rate to carbapenems which exemplify the increasing trend of resistance level (16). The resistance pattern varies depending on whether the isolate belongs to A. baumannii or non-baumannii Acinetobacter species. Studies have demonstrated that the rate of carbapenem resistance is more in A. baumannii group as compared to non-baumannii Acinetobacter. In the present study $53.3 \%$ of the $A$. baumannii isolates were sensitive to carbapenems wherein 72.7\% of the non-baumannii Acinetobacter isolates were sensitive to carbapenems. In concordance with this, Shareek et al. (17) reported $25 \%$ and $73 \%$ sensitivity of $A$. baumannii and non-baumannii Acinetobacter species to carbapenems, respectively. Study by Jaggi et al. (10) also reported high resistance rate (90\%) of the A. baumannii species towards carbapenems (10). Additionally, study by Nazmul et al. (12) in Malaysia revealed as high as $92.5 \%$ resistance of Acinetobacter species to meropenem. Besides this, a recent study from India has shown 50\% sensitivity of Acinetobacter species to carbapenems (18).

In this study, $80 \%$ of the $A$. baumannii isolates were sensitive to tigecycline. Shareek et al. (17) from India reported $61.4 \%$ sensitivity of $A$. baumannii to tigecycline. Furthermore, Van et al. (15) from Vietnam reported 58.7\% susceptibility to tigecycline.

In this study colistin was the only drug that showed $100 \%$ sensitivity against all the species of Acinetobacter. Likewise, Van et al. (15) also reported 100\% sensitivity to colistin. Additionally, Jaggi et al. (10) reported around $1.2 \%$ resistance and Rani et al. (18) reported $80 \%-90 \%$ sensitivity to Colistin. Correspondingly, Vakili et al. (11) from Iran reported $11.6 \%$ resistance to colistin.

Colistin and tigecycline remain the only active antibiotics for the treatment of MDR A. baumannii. Tigecycline has a large volume of distribution resulting in a low serum peak concentration and a suboptimal clinical outcome. Breakthrough bacteremia during tigecycline therapy can be observed in drug resistant $A$. baumannii infection. A study done by Kim et al. (19) demonstrated that the efficacy of tigecycline-based therapy was comparable to that of colistin-based therapy in patients with multidrug-resistant and extensively drugresistant $A$. baumannii. The same study also revealed a trend toward higher clinical and microbiological success rates and lower 30day, ICU, and in-hospital mortality rates in the combination therapy group as compared to 
monotherapy. Colistin, is a narrow spectrum cationic lipopeptide rapidly bactericidal against gram-negative bacteria. Moreover, colistin administration alone is associated with significant nephrotoxicity and hetero-resistance in MDR A. baumannii clinical isolates. A recent (2015) meta-analysis suggested that colistin is probably as safe and efficacious as standard antibiotics for the treatment of drug-resistant $A$. baumannii infection (20).

Thus, new alternative antibiotics or treatment options with newer combinations is the need of the hour for successful management of multidrug-resistant $A$. baumannii, until then combination therapies including tigecycline; colistin is a reasonable approach.

\section{Conclusion}

Injudicious use of antibiotics has led to the development of multidrug-resistant $A$. baumannii species which make therapeutic decisions to be challenging.

In the present study high rate of resistance was observed to broad-spectrum cephalosporin, aminoglycosides, fluoroquinolones and combination of penicillin/beta-lactamase inhibitor. Colistin was found to be the most effective drug (100\% sensitivity) for all species of Acinetobacter. For A. baumannii colistin was the most effective drug followed by tigecycline and carbapenemes.

\section{Authors' Contributions}

Conception and design: RT, DC, MD

Analysis and interpretation of the data: RT, DC Drafting of the article: RT, DC, RW, SD, MD

Critical revision of the article for important intellectual content: RT, DC, MD

Final approval of the article: RT, DC, RW, SD, MD

Provision of study materials or patients: RW, SD

Collection and assembly of data: RW, SD

\section{Correspondence}

Dr Deepti Chopra

DTCD (University of Delhi), MD (University of Delhi)

Associate Professor

Department of Pharmacology,

Government Institute of Medical Sciences,

Kasna, Greater Noida,

Uttar Pradesh-201310, India.

Tel: 919818710237

E-mail: drdeeptichopra@yahoo.co.in

\section{References}

1. Van Looveren M, Goossens H. Antimicrobial resistance of Acinetobacter spp. in Europe. Clin Microbiol Infect. 2004;10(8):684-704. https:// doi.org/10.1111/j.1469-0691.2004.00942.x

2. Rice LB. Federal funding for the study of antimicrobial resistance in nosocomial pathogens: no ESKAPE. $J$ Infect Dis. 2008;197(8):10791081 https://doi.org/10.1086/533452

3. Bergogne-Berezin E, Towner KJ. Acinetobacter spp. as nosocomial pathogens: microbiological, clinical, and epidemiological features. Clin Microbiol Rev. 1996;9(2):148-165.

4. Maragakis LL, Perl TM. Acinetobacter baumannii: epidemiology, antimicrobial resistance, and treatment options. Clin Infect Dis. 2008;46(8):1254-1263. https://doi.org/ $10.1086 / 529198$

5. Bouvet PJ, Grimont PA. Identification and biotyping of clinical isolates of Acinetobacter. Ann Inst Pasteur Microbiol. 1987:138(5):569-578.

6. Clinical and Laboratory Standards Institute. Performance standards for antimicrobial susceptibility testing; 2oth informational supplement. CLSI document M1Oo-S2O. Wayne, PA: Clinical and Laboratory Standards Institute; 2010.

7. Falagas ME, Koletsi PK, Bliziotis IA. The diversity of definitions of multidrug-resistant (MDR) and pandrug-resistant (PDR) Acinetobacter baumannii and Pseudomonas aeruginosa. $J$ Med Microbiol. 2006;55:1619-1629. https://doi. org/10.1099/jmm.0.46747-0

8. Lahiri KK, Mani NS, Purai SS. Acinetobacter spp as nosocomial pathogen: clinical significance and antimicrobial sensitivity. MJAFI. 2004;60(1):7-10. https://doi.org/10.1016/ So377-1237(04)80148-5

9. Villers D, Espase E, Coste-Burel M, Giauffret F, Ninin E, Nicolas F, et al. Nosocomial Acinetobacter baumannii infections: microbiological and clinical epidemiology. Ann Intern Med. 1998;129(3):182-189. 
10. Jaggi N, Sissodia P, Sharma L. Acinetobacter baumannii isolates in a tertiary care hospital: antimicrobial resistance and clinical significance. JMID. 2012;2(2):57-63. https://doi.org/10.5799/ ahinjs.02.2012.02.0043

11. Vakili B, Fazeli H, Shoaei P, Yaran M, Ataei B, Khorvash F, et al. Detection of colistin sensitivity in clinical isolates of Acinetobacter baumannii in Iran. J Res Med Sci. 2014;19(Suppl 1):S67-S70.

12. Nazmul MHM, Jamal H, Fazlul MKK. Acinetobacter species-associated infections and their antibiotic susceptibility profiles in Malaysia. Biomed Res-India. 2012;23(4):571-575. https://pdfs.semanticscholar.org/a14d/49d62327 o53ca54e330205b82000262f54ee.pdf

13. Shakibaie MR, Adeli S, Salehi MH. Antibiotic resistance patterns and extended spectrum b-lactamase production among Acinetobacter spp. isolated from an intensive care unit of a hospital in Kerman, Iran. Antimicrob Resist Infect Control. 2012;1:1. https://doi.org/10.1186/20472994-1-1

14. Gupta N, Gandham N, Jadhav S, Mishra RN. Isolation and identification of Acinetobacter species with special reference to antibiotic resistance. J Nat Sci Biol Med. 2015;6(1):159162. https://doi.org/10.4103/o976-9668.149116

15. Van TD, Dinh QD, Vu PD, Nguyen TV, Pham C V, Dao TT, et al. Antibiotic susceptibility and molecular epidemiology of Acinetobacter calcoaceticus-baumannii complex strains isolated from a referral hospital in northern Vietnam. $J$ Glob Antimicrob Resist. 2014;2(4):318-321. https://doi.org/10.1016/j. jgar.2014.05.003
16. Gaur A, Garg A, Prakash P, Anupurba S, Mohapatra TM. Observations on carbapenem resistance by minimum inhibitory concentration in nosocomial isolates of Acinetobacter species: an experience at a tertiary care hospital in North India. $J$ Health Popul Nutr. 2008;26(2):183188. https://www.ncbi.nlm.nih.gov/pmc/articles/ PMC2740671/

17. Shareek PS, Sureshkumar D, Ramgopalakrishnan, Ramasubramanian V, Ghafur KA, Thirunarayanan MA. Antibiotic sensitivity pattern of blood isolates of Acinetobacter species in a tertiary care hospital: a retrospective analysis. $A m$ $J$ Infect Dis. 2012;8(1):65-69. http://thescipub. com/PDF/ajidsp.2012.65.69.pdf

18. Rani P, Latha MB, Reddy SG, Bilolikar AK. A study of Acinetobacter from various clinical specimens and its antibiotic sensitivity pattern in a tertiary care hospital. $J$ Med Sci Res. 2015;3(4):162-165. https://doi.org/10.17727/ JMSR

19. Kim W-Y, Moon J-Y, Huh JW, Choi S-H, Lim C-M, Koh Y, et al. Comparable efficacy of tigecycline versus colistin therapy for multidrugresistant and extensively drug-resistant Acinetobacter baumannii pneumonia in critically ill patients. PLoS One. 2016;11(3):e0150642. https://doi.org/10.1371/journal.pone.0150642

20. Chen Z, Chen Y, Fang Y, Wang X, Chen Y, Qi Q, et al. Meta-analysis of colistin for the treatment of Acinetobacter baumannii infection. Sci Rep. 2015;5:17091. https://doi.org/10.1038/srep17091 